\title{
L'Association Française pour l'Etude des Irrigations et du Drainage (AFEID)
}

\author{
par Jean Cothenet \\ Ingénieur général honoraire du GREF \\ Président d'honneur de l'AFEID
}

C'est en 1950 qu'a été constituée la Commission Internationale des Irrigations et du Drainage (CIID).

Son secrétariat général étant installé à New Delhi, il était dans la logique des choses que le $1^{\text {er }}$ Congrès International se déroulât à New Delhi (1951) autour de deux questions très générales :

- Bilan national du développement et de la pratique des irrigations.

- Problèmes actuels relatifs aux irrigations et au drainage.

De retour de l'Inde, la délégation française, fortement impressionnée par ce premier contact, décidait la création de l'AFEID (1952) et présentait la candidature de la France pour organiser le $2^{\mathrm{e}}$ Congrès International des Irrigations et du Drainage (Alger, 1954). CIID.

Ainsi naquit l'AFEID, comité national français de la

\section{Buts et objets de l'association}

Ces origines vont conduire l'AFEID à garder présentes trois préoccupations majeures:

- promouvoir le développement des applications de l'hydraulique agricole en France,

- favoriser la diffusion vers l'étranger des techniques françaises en ce domaine,

- contribuer aux efforts de coopération technique internationale.

\subsection{Développer l'hydraulique agricole en France}

La maîtrise de l'eau par l'agriculteur est une nécessité qui s'impose d'autant plus que l'activité agricole est soumise à de multiples contraintes sur le plan de l'efficacité, de la qualité des produits, des prix, sans parler des contraintes environnementales.

Cela explique l'importance des investissements consentis par les exploitants agricoles dans les décennies précédentes, jusqu'à ce que les conséquences de la dernière réforme de la politique agricole commune n'entraînent la chute brutale et sans doute momentanée de leurs investissements.

Sur ce plan l'AFEID ne veut pas se considérer comme un groupe de pression vis-à-vis des Pouvoirs Publics. Pour évi- ter toute ambiguité, elle a modifié son vocabulaire et remplacé l'expression «développement de l'hydraulique agricole » par sa «maîtrise raisonnée ».

Dans cet esprit, elle participe activement à l'élaboration de règles de bonne conduite, dans le cadre de son Comité Technique et des Groupes de Travail qu'il anime. Ces derniers rassemblent les meilleurs experts français sur des thèmes qui répondent aussi bien aux préoccupations françaises qu'à celles qui apparaissent au sein de la CIID.

\subsection{Favoriser la diffusion vers l'étranger des techniques françaises}

Pour favoriser la diffusion vers l'étranger des techniques françaises, plusieurs moyens sont à notre disposition :

- la participation aux groupes de travail de la CIID. Ainsi que l'a montré Henri Tardieu, l'AFEID essaye d'avoir un représentant dans chaque groupe de travail. De ce fait, la délégation française se fait généralement remarquer par sa présence nombreuse et efficace dans les différentes manifestations de la CIID

- les congrès internationaux, organisés tous les 3 ans,

- la rédaction d'ouvrages techniques et leur diffusion par et sous le label de la CIID.

On citera entre autres la rédaction de deux chapitres du Dictionnaire technique de la CIID et la publication d'ouvrages tels que :

- "Maintenance des périmètres irrigués" (J. Verdier et $\mathrm{J}$. Millo, 1992).

- «Critères de choix des systèmes d'irrigation» (H. Hlavek, 1993).

- «Automatisation des réseaux d'irrigation en canaux 》 (J. Goussard, 1993),

ouvrages qui ont été traduits en anglais et largement diffusés.

1.3 Contribuer aux efforts de coopération technique internationale

Dans le cadre de la CIID ou sous l'impulsion du ministère des Affaires Etrangères ou du ministère de la Coopération, 
l'AFEID a été amenée à apporter son expérience et son savoir-faire dans le cadre de projets précis.

A la demande de la CIID sous l'égide de l'Africa Focus Group, avec l'appui financier du ministère de la Coopération et en synergie avec la FAO et la Banque Mondiale, nous avons engagé le processus de création d'une Association Régionale pour l'Irrigation et le Drainage - dit projet "ARID»- visant à regrouper les pays de l'Afrique de l'ouest au sud du Sahara qui, bien que connaissant un fort développement de l'irrigation, n'ont pas ou n'ont plus, à l'exception du Nigéria, de comités nationaux actifs.

Plus récemment, à la demande de la CIID mais aussi à celle du ministère de l'Agriculture et du ministère des Affaires Etrangères, des relations privilégiées se sont établies avec le Maroc

Ajoutons que l'AFEID a joué un rôle déterminant dans la sensibilisation des ministères français concernés et des autres associations françaises de l'eau, à la création du futur Conseil Mondial de l'Eau et qu'elle n'a pu que se réjouir de voir le siège de ce Conseil s'installer à Marseille.

\section{2. Moyens de l'association}

A l'origine, l'association présentait une forte dominante d'ingénierie: fonctionnaires de l'agriculture et des travaux publics, bureaux d'études, sociétés d'aménagement étaient les principaux partenaires avec une forte présence de l'administration.

Ainsi la cheville ouvrière du Congrès d'Alger (1954) fut le Service de l'Hydraulique et de la Colonisation dirigée par M. Droulin, tandis que celle du Congrès de Grenoble (1981) fut le Service de l'Hydraulique Agricole dirigé par $M$. Darves-Bornoz, assisté de M.G. Manuellan. Aujourd'hui, ainsi que le montre brillamment Henri Tardieu, l'approche ne peut être que globale. L'AFEID est parfaitement consciente de cette nécessité. La composition de son Conseil d'Administration illustre cette préoccupation : rassembler l'ensemble de la filière, de la recherche jusqu'aux usagers.

Les ministères intéressés par son activité sont invités en qualité d'observateurs: Agriculture, Environnement, Affaires étrangères, Coopération, Recherche.

La composition des groupes de travail reflète le même souci, ce qui a conduit lorsque cela était utile, à constituer des groupes mixtes AFEID-RNED.HA $\left({ }^{\prime}\right)$.

Enfin, en dehors des assemblées générales qui sont l'occasion de mieux connaitre nos adhérents, l'association organise des journées techniques en partenariat avec la profession agricole :

- en 1994, les journées d'Orléans, avec la Chambre régionale d'Agriculture du Centre, sur le thème «Irrigation, environnement et gestion des ressources en eau »,

- en 1995, les journées de Toulouse, avec la Chambre régionale d'Agriculture de Midi-Pyrénées sur le thème " $L a$ gestion de l'eau dans les exploitations de grande culture", - en 1996, les journées de Colmar, avec la Chambre régionale d'Agriculture d'Alsace sur le thème "Irrigation et drainage dans le contexte économique et environnemental actuel ».

(1) Réseau national d'expérimentation et de démonstration hydraulique agricole.

\begin{tabular}{|c|c|c|c|}
\hline \multicolumn{4}{|c|}{ Conseil d'Administration de l'AFEID au 10 mai 1996} \\
\hline \multicolumn{4}{|c|}{ Bureau : } \\
\hline Président & M. TARDIEU & Henri & CACG \\
\hline \multicolumn{4}{|l|}{ Vice-Président } \\
\hline Président du Comité Technique & M. RIEUL & Léopold & CEMAGREF \\
\hline \multirow[t]{3}{*}{ Vice-Présidents } & M. JOYES & Michel & CGGREF \\
\hline & M. PLANTEY & Jacques & $\mathrm{SCP}$ \\
\hline & M. MASSE & Jack & ITCF \\
\hline Secrétaire Général & M. LACROIX & François & CEMAGREF \\
\hline \multicolumn{4}{|c|}{ Administrateurs : } \\
\hline \multirow[t]{14}{*}{ a) Organismes: } & & Représenté par & \\
\hline & AFGR & M. SOUTY & Jean-Claude \\
\hline & Agence Eau Loire-Bretagne & M. DUBOIS de LA SABLONIËRE & François \\
\hline & APCA & M. de DEMANDOLX-DEDONS & José \\
\hline & CIRAD-CA & M. PAGES & Jacques \\
\hline & ENSA Rennes & M. DUCHESNE & \\
\hline & FNCUMA & M. VAQUIE & Pierre-F. \\
\hline & ORSTOM & M. RIEU & Michel \\
\hline & OTECH SA & M. HISTEL & Michel \\
\hline & BRL & M. NICOL & Jean-Pierre \\
\hline & CNR & M. GUILHAUDIN & Patrick \\
\hline & EDF - Direction Equipement & M. COURIER & Michel \\
\hline & INRA & M. PERRIER & Alain \\
\hline & Ordre des Géomètres & M. LAGOUTTE & Michel-F. \\
\hline \multicolumn{4}{|l|}{ 2) Adhérents } \\
\hline & M. FAIVRE & André & \multirow{6}{*}{$\begin{array}{l}\text { Ass. Dép. Agricole } \\
\text { CDA Charente } \\
\text { Maritime } \\
\text { ENGREF }\end{array}$} \\
\hline & M. GARRES & Bernard & \\
\hline & M. MAROTEIX & Jacques & \\
\hline & M. DELACOURT & Alain & \\
\hline & M. COLIN de VERDIĖRE & Michel & \\
\hline & M. MESNY & Michel & \\
\hline
\end{tabular}


Parallèlement, l'association a souhaité développer des relations plus étroites avec les autres associations française de l'eau. Dans le passé il a été possible d'organiser des journées techniques sur des thèmes communs, tels que "Irrigation 2000 » avec la SHF et «Les petits barrages en milieu rural » avec le CFGB.

Plus récemment, la SHF et l'AFEID ont provoqué des réunions d'information avec les autres associations françaises de l'eau qui ont abouti à la mise sur pied d'un Comité de Liaison des Associations Françaises de l'Eau, le CLAFE. L'objectif est que tous les partenaires puissent bénéficier d'une bonne information sur les initiatives internationales concernant le domaine de l'eau et Dieu sait si elles sont nombreuses actuellement.

\section{NONCLUSION}

Sur le plan mondial, ainsi que l'a montré la FAO dans un chapitre spécial de son rapport annuel 1993 consacré au thème "Politiques de l'eau et agriculture", celle-ci est confrontée à un double défi : produire plus pour nourrir une population toujours plus nombreuse, tout en réduisant sa consommation d'eau, afin de permettre la satisfaction d'autres besoins prioritaires en particulier ceux de l'industrie et des villes.

En France, le défi n'est pas fondamentalement différent. Notre agriculture est aujourd'hui le troisième préleveur d'eau, derrière les centrales thermiques et les collectivités locales, mais elle est le premier consommateur net. Cette situation a créé et créera encore des difficultés dans certaines zones du territoire, notamment si les prélèvements se font aux dépens des débits d'étiages ou de nappes souterraines insuffisamment puissantes, au lieu de mobiliser des eaux hivernales stockées dans des réservoirs naturels ou artificiels.

C'est dire l'importance d'une gestion globale, telle que la définit la loi sur l'eau. Cette approche condamne le gaspillage et oblige les irrigants à une solide maîtrise de leurs pratiques. De la même façon, les concepteurs ne peuvent ignorer l'impact de ces travaux sur l'environnement et doivent, par conséquent, en intégrer tous les aspects lors de l'élaboration de leurs projets.

C'est l'ambition de l'AFEID et de tous ses adhérents d'apporter leur contribution à une meilleure maîtrise de l'eau en agriculture. 